\title{
Identification and in silico analysis of 14 novel GJB1, MPZ and PMP22 gene mutations
}

\author{
Gabriel Miltenberger-Miltenyi ${ }^{1}$, Thomas Schwarzbraun ${ }^{2}$, Wolfgang N Löscher ${ }^{3}$, \\ Julia Wanschitz ${ }^{3}$, Christian Windpassinger ${ }^{2}$, Hans-Christoph Duba ${ }^{4}$, Rainer Seidl ${ }^{5}$, \\ Gerhard Albrecht $^{6}$, Helga Weirich-Schwaiger ${ }^{1}$, Heinz Zoller ${ }^{7}$, Gerd Utermann ${ }^{1}$, \\ Michaela Auer-Grumbach ${ }^{2}$ and Andreas R Janecke*,1
}

\footnotetext{
${ }^{1}$ Division of Clinical Genetics, Innsbruck Medical University, Innsbruck, Austria; ${ }^{2}$ Institute of Human Genetics, Medical University Graz, Graz, Austria; ${ }^{3}$ Clinical Department of Neurology, Innsbruck Medical University, Innsbruck, Austria; ${ }^{4}$ Department for Human Genetics, General Women and Children Hospital, Linz, Austria; ${ }^{5}$ Department of Pediatrics, Wien Medical University, Wien, Austria; ${ }^{6}$ Department of Neurology, Kaiser-Franz-Josef-Spital, Wien, Austria;

${ }^{7}$ Department of Gastroenterology and Hepatology, Innsbruck Medical University, Innsbruck, Austria
}

Duplication within the chromosome 17p11.2 (CMT1Adup), peripheral myelin protein 22 (PMP22), myelin protein zero (MPZ) and gap junction $\beta 1$-protein (GJB1) gene mutations are frequent causes of the CharcotMarie-Tooth disease (CMT). A large number of mutations in these genes are listed in databases. Sequence variants identified in patients are frequently reported as mutations without further evaluation. We analyzed 250 consecutively recruited unrelated Austrian CMT patients for CMT1Adup by microsatellite marker typing, real-time PCR or MLPA, and found 79 duplications (31.6\%). The coding regions of the PMP22, MPZ and GJB1 genes were analyzed by direct sequencing in the remaining patients; 28 patients showed mutations, 14 of which were novel. We scored the pathogenicity of novel missense mutations by segregation studies and by their exclusion in control samples. Our comprehensive literature study found that up to $60 \%$ of the reported mutations in these genes had not been evaluated regarding their pathogenicity, and the PANTHER bioinformatics tool was used to score novel and published missense variants. The PANTHER program scored known polymorphisms as such, but scored $\sim 82-88 \%$ only of the published and novel mutations as most likely deleterious. Mutations associated with axonal CMT were less likely to be classified as deleterious, and the PMP22 S72L mutation repeatedly associated with severe CMT was classified as a polymorphism using default parameters. Our data suggest that this in silico analysis tool could be useful for assessing the functional impact of DNA variations only as a complementary approach. The CMT1Adup, GJB1, MPZ and PMP22 mutation frequencies were in the range of those described in other CMT patient collectives with different ethnical backgrounds.

European Journal of Human Genetics (2009) 17, 1154-1159; doi:10.1038/ejhg.2009.29; published online 4 March 2009

Keywords: hereditary motor and sensory neuropathy; phenotype-genotype correlation; CMT1; CMT2; nerve conduction velocity; bioinformatics

\footnotetext{
*Correspondence: Dr AR Janecke, Division of Clinical Genetics, Innsbruck Medical University, Schoepfstrasse 41, A-6020 Innsbruck, Austria. Tel: + 435129003 70542; Fax: + 435129003 73510; E-mail: Andreas.Janecke@i-med.ac.at Received 13 March 2008; revised 19 January 2009; accepted 22 January 2009; published online 4 March 2009
}

\section{Introduction}

The Charcot-Marie-Tooth disease (CMT; MIM 118220), also known as hereditary motor and sensory neuropathy, occurs with an estimated frequency of 1:2500 individuals. ${ }^{1}$ The main clinical characteristics are slowly progressive distal muscle weakness and wasting first of the lower and later 
usually also of the upper limbs, sensory loss and foot deformity. CMT has been subdivided according to the nerve electrophysiological criteria into two main groups: $:^{2-4}$ CMT1, which is characterized by reduced motor nerve conduction velocities (MNCV) of the median nerve (MNCV $<38 \mathrm{~m} / \mathrm{s}$ ) and nerve demyelination, and the axonal variant, CMT2, with MNCV being almost normal (MNCV $>38 \mathrm{~m} / \mathrm{s}$ ), but with reduced amplitudes of compound motor-evoked potentials. An additional classification of intermediate CMT has been suggested in cases with MNCV between 25 and $45 \mathrm{~m} / \mathrm{s}^{5}$ CMT1 is frequently associated with duplications on the chromosome $17 \mathrm{p} 11.2-$ p12 (CMT1A), which contains the peripheral myelin protein 22 gene (PMP22; MIM 601097). ${ }^{6}$ Further mutations in 36 genes have been described in CMT patients (http:// www.molgen.ua.ac.be/CMTMutations/

default.cfm). Mutations in the gap junction $\beta$-1 protein located on chromosome Xq13.1 (GJB1 or Connexin 32, Cx32; MIM 304040) may lead to CMT type X1 (CMTX1), and electrophysiology may suggest both demyelinating and/or axonal nerve damage, thus resulting in either a CMT1, CMT2 or in an intermediate phenotype. ${ }^{7}$ Myelin protein zero (MPZ or P0; MIM 159440) mutations are associated with CMT1, but can also cause CMT2 or the Dejerine-Sottas syndrome (DSS) ${ }^{8,9}$ The PMP22 mutations can cause CMT1, DSS, congenital hypomyelination (CHN) and, if inactivated, can also cause hereditary neuropathy with a liability to pressure palsies (HNPP). ${ }^{10}$

We examined 250 unrelated Austrian CMT patients for the CMT1A duplication 17p11.2-p12. A total of 171 individuals without duplication were screened for mutations in the GJB1, $M P Z$ and PMP22 genes. We report on the mutation frequency distribution in this population, and describe 14 novel sequence variations detected in patients. The pathogenicity of novel and published mutations was also evaluated using the recent bioinformatics program, PANTHER. ${ }^{11}$

\section{Patients, materials and methods Patients}

Patients diagnosed with CMT were referred from neurological departments throughout Austria, and were consecutively included in this study. Written informed consent was obtained from all the tested persons according to the Declaration of Helsinki. Most of the patients were of Austrian ethnicity. The clinical criteria of CMT required at least the presence of distinct gait abnormalities, because of weakness of the foot extensors. On the basis of the MNCV measurements, 85 patients were classified as CMT1, and 29 patients as CMT2. In the remaining cases, the clinical data were incomplete for further subclassification.

\section{Mutation analysis}

DNA was extracted from peripheral blood samples using an automated extractor according to the manufacturer's protocols (GenoM 48, Qiagen, Vienna, Austria). Samples were analyzed for the presence of a 1.4-Mb CMT1A duplication on chromosome $17 \mathrm{p}$ by genotyping either five polymorphic microsatellite markers (D17S2220, D17S2224, D17S2226, D17S2227 and D17S2230), ${ }^{12}$ real-time PCR or multiplex ligation-dependent probe amplification (SALSA MLPA kit P033(B)). The coding regions and splice sites of the GJB1, MPZ and PMP22 genes were amplified by PCR in the index patients (Supplementary Table 1). After the detection of a mutation in a proband, a number of clinically affected or healthy relatives were tested for this mutation, when available. The sequencing reactions were analyzed on the ABI 3100 DNA sequencer, using the BigDye terminator mix (Applied Biosystems, Vienna, Austria). PCR amplicons from anonymous healthy controls were analyzed for the presence of novel mutations, using denaturing high performance liquid chromatography.

\section{Bioinformatics analysis}

We used the in silico sequence alignment program, ClustalW, and the evolutionary model program, PANTHER, ${ }^{11}$ to estimate the functional consequences of published and novel missense mutations in these genes (http:// www.pantherdb.org/). The mutation nomenclature is based on protein reference sequences, such as MIM NP_000157 (GJB1) and MIM NP_000295 (PMP22). Consistent with the CMT mutation database (http://www.molgen.ua.ac.be/ CMTMutations/default.cfm), the nomenclature describing MPZ mutations was based on the MPZ sequence D10537 (http://www.ncbi.nlm.nih.gov/entrez/), as published by Hayasaka et al, ${ }^{13}$ and not on the MPZ RefSeq NM_000530 (http://www.ncbi.nlm.nih.gov/entrez/).

\section{Results and discussion}

Among the 250 unrelated CMT patients, 79 of them (31.6\%) harbored the CMT1A duplication. Of these, 43 patients were classified as CMT1, and the MNCV data were lacking for 36 patients. One patient was identified to harbor the typical HNPP deletion.

A subsequent mutation analysis of GJB1, MPZ and PMP22 in CMT patients without CMT1Adup revealed 28 heterozygous or hemizygous mutations. We identified 4 novel truncating or frameshifting, and 10 novel missense mutations, which we considered pathogenic (Table 1). Neither of these mutations was detected among the 93 controls (blood donors).

Disease onset was reported in the first to third decade of life in all patients with novel mutations, except for a female with the MPZ mutation, p.D224Y. Four of six patients with the MPZ mutations were classified as CMT1. The MPZ mutations, p.I30S and p.R185AfsX66, were associated with a severe, early-onset demyelinating phenotype similar to those observed earlier in patients with mutations of codon 30, and the abnormal C-terminal 


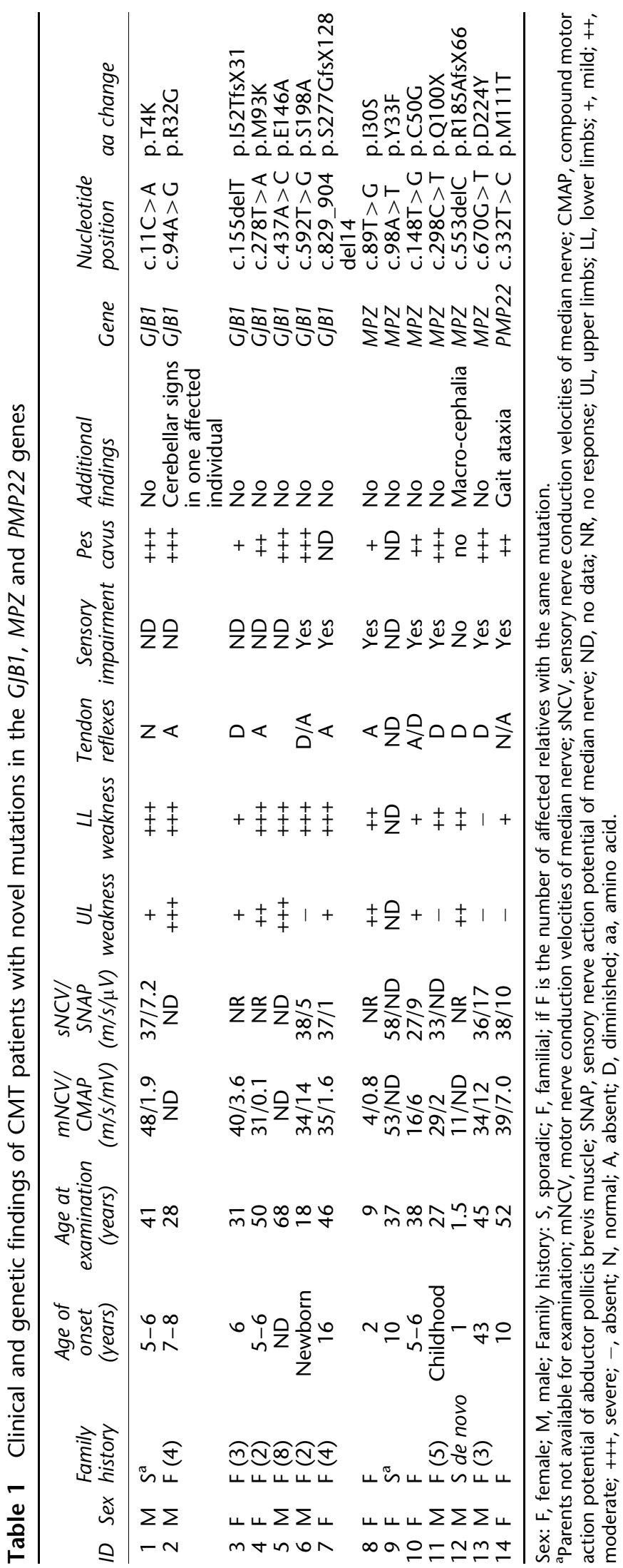

p.R185AfsX66 protein $^{14,15}$ further confirming the observed genotype-phenotype correlation of the MPZ mutations. ${ }^{8}$

One CMTX patient additionally showed cerebellar ataxia, the significance of which is unclear at present. Ataxia did not segregate within the family, and the most common genetic causes of ataxia (Friedreich ataxia, SCA 1, 2, 3 and 6) were excluded. The individual carrying the novel PMP22 mutation had median MNCV of $39 \mathrm{~m} / \mathrm{s}$ and gait ataxia.

The identified mutations: p.R22Q, p.R75W, p.W77S, p.R107W, p.R142Q, p.V181M and p.N205S in GJB1; p.S20F, p.D60H, p.I62M and p.S78L in MPZ; and pS72L, c.281-282insG and p.T118M in PMP22 were described earlier and are listed in the European CMT Consortium Mutations Update database, accessed at http://www. molgen.ua.ac.be/CMTMutations/default.cfm.

\section{Truncating and frameshifting mutations}

The GJB1 p.I52TfsX31 mutation causes an early truncation of the 283-amino acid wild-type protein. The p.Q100X in exon 3 of MPZ most likely causes a nonsense-mediated decay, and segregates with the disease in a large pedigree. The c.553delC deletion in exon 4 of MPZ results in a frameshift with a large abnormal C-terminal peptide sequence (p.R185AfsX66). This mutation was found to occur de novo in our patient. An MPZ mutation, c.554delG, with the same consequence at the protein level has been described as a cause of CMT earlier. ${ }^{15}$ The deletion of 14 nucleotides, c.829_843, in GJB1 removes the last 6 amino acids and adds 128 abnormal amino acids $\mathrm{C}$ terminally (p.S277GfsX128), and is most likely to interfere with the normal connexin structure.

\section{Missense mutations}

The high degree of conservation of the identified mutated residues in GJB1, MPZ and PMP22 is represented in Figure 1, and these missense changes are non-conservative (Table 1). Distinct mutations within three mutated GJB1 codons, p.S198P, p.M93V and p.E146K, have been described earlier in CMT patients. ${ }^{16}$ Notably, the MPZ mutation, p.C50G, is predicted to cause loss of a disulfide bridge in MPZ. ${ }^{17}$ The MPZ mutation, p.I30S, affects the highly conserved, first amino acid residue after the cleavage of a 29 amino acid signal peptide. Deletion of isoleucin-30, as well as changes p.I30T and p.I30M have been described in patients with CMT1 and DSS. However, family studies regarding the MPZ mutation, p.Y33F, and the GJB1 mutation, p.T4K, were not feasible.

The generally used criteria for scoring the pathogenicity of identified missense variants are the absence of identified sequence variants among healthy controls and the segregation of mutations with the disease within families. In addition, co-investigated patient cohort samples are usually considered to serve as controls for determining the frequency of mutations. However, especially in simplex 
a

Homo sapiens/NP_000157

Rattus norvegicus/NP_058947

Mus musculus/NP_032150

Equus caballus/NP 001075360

Bos taurus/NP_776494

Xenopus tropicalis/NP_001001240

Homo sapiens/ NP_000157

Rattus norvegicus/ NP 058947

Mus musculus/ NP_032150

Equus caballus/ NP 001075360

Bos taurus/ NP_776494

Xenopus tropicalis/ NP 001001240

Homo sapiens/ NP_000157

Rattus norvegicus/ NP_058947

Mus musculus/ NP_032150

Equus caballus/ NP 001075360

Bos taurus/ NP_776494

Xenopus tropicalis/ NP 001001240

Homo sapiens/ NP_000157

Rattus norvegicus/ NP_058947

Mus musculus/ NP_032150

Equus caballus/ NP 001075360

Bos taurus/ NP_776494

Xenopus tropicalis/ NP 001001240

b

Homo sapiens/ NP 000521

Rattus norvegicus/ NP_058723

Mus musculus/ NP_032649

Equus caballus/ NP 001075363

Canis familiaris/ENSCAFP00000019099

Bos taurus MYPO/ ENSBTAP00000045141

Homo sapiens/ NP 000521

Rattus norvegicus/ NP_058723

Mus musculus/ NP_032649

Equus caballus/ NP_001075363

Canis familiaris/ENSCAFP00000019099

Bos taurus MYPO/ ENSBTAP00000045141

Homo sapiens/ NP_000295

Rattus norvegicus/ NP_058733

Mus musculus/ NP_032911

Equus caballus/ NP_001075405

Danio rerio/ NP 958468

Canis familiaris/ENSCAFP00000026442 Gallus gallus/ ENSGALP00000002076

\section{4}

32

MNVTTLYTLLSGVNRHSTAIGRVWLSVIFIFRIMVLVVAA MNWT GLYTLLSGVNRHSTAIGRVWLSVIF IFRIMVLVVAA MNWT TLYTLLSGVNRHSTAIGRVWLSVIF IFR IMVLVVAA MNW T GL YTLLS GVNRHSTAIGRVWLSVIF IFR IMVLVVAA MNW T GLYTLLS GVNRHSTAIGRVWLSVIF IFR IMVLVVAA MNW A GLYAILSGVNRHSTSIGRIWLSVVF IFRIMVLVVAA 93

NSVCYDQFFPISHVRLWSLQLILVSTPALLVAM IVAHQQH NSVCYDHFFPISHVRLWSLQLILVSTPALLVAMFVAHQQH NSVCYDHFFPISHVRLWSLQLILVSTPALLVAMFVAHQQH NSVCYDHFFPISHVRLWSLQLILVSTPALLVAMFVAHQQH NSVCYDHFFPISHVRLWSLQLILVSTPALLVAMFVAHQQH NSVCYDHFFPISHIRLWALQLIIVSTPALLVAMFVAHLQH 146

HGDPLHLEEVKRHKVHISGTLWWTYVISVVFRLLF国VFM HGDPLHLEEVKRHKVHISGTLWWTYVISVVFRLLFEAVFM HGDPLHLEEVKRHKVHISGTLWWTYVISVVFRLLFEAVFM HGDPIHLEEVKRHKVHISGTLWWTYVISVVFRLLFEAAFM HGDPLHLEEVKRHKVHISGTLWWTYVISVVFRLLFEAAFM HVKDQELAEVKKHKVKISGTLWWTYIISVFFRIIFEAAFM 198

AMVRLVKCDVYPCPNTVDCFVSRPTEKTVFTVFMLAA SGI AMVRLVKCEAF PCPNTVDCFVSRPTEKTVFTVFMLAASGI AMVRLVKCEAFPCPNTVDCFVSRPTEKTVFTVFMLAA SGI AMVRLVKCDAYPCPNTVDCFVSRPTEKTVFTVFMLAA SGI AMVRLVKCDAYPCPNTVDCFVSRPTEKTIFTVFMLAA SGI SMIRLVKCDAYPCPNTVDCFVSRPTEKTIFTVFMLVA SGF

3033

AVLLFSSLVLSPAOATVYYTDREVHGAVGSRVTL AALLFSSLVLSPTLA IVVYTDREVYGAVRSQVTLFC AALLF SSLVLSPALA IVVYTDREIYGAVGSQVTLFC AALLF SSLVLSPAQA I VVYTDKEVYGAVGSRVTLHC AVLLFSSLVLPPAQA IVVYIPDREVHGAVGSRVTLHC AALLFSSLVLSPVQA IVUYTDKEVHGAVGSQVTLYC 224

SAMEKGKLHKPGKDASKRGRQTPVLYAMIDHSRSTK SAMEKGKFHKSSKDSSKRGRQTPVLYAMI DESRSTK SAMEKGRFHKSSKDSSKRGRQTPVLYAMI DHSRSTK SAMEKGKLHKPGKDTSKRGRQTPVLYAMI DESRSTK SAMEKGKLHKAGKDTSKRGRQTPVLYAMI DESRSTK SAMEKGKLHKTAKDASKRGRQTPVLYAMI DHSRSTK

111

IFQILAGLCYMSAAAIYTVRHPEWHLNSD VFQILAGLCVMSAAAIYTVRHSEWHVNND FFQILAGLCVMSAAAIYTVRHSEWHVNTD IFQILAGLCVMSAAS IYTVRHPEWHLDSA VFQILASLFYMSGAIIYTVMSPQWKNDSD VFQILAGLCVMSAASIYTVRHPEWHFNSD IFQILAGLCVMSGAAIFTVRHTDWHEASE

Figure 1 Conservation of mutated amino acids according to a ClustalW sequence alignment. (a) GJB1 (MIM NP_000157), (b) MPZ (MIM NP_000521) and (c) PMP22 (MIM NP_000295). Mutated residues are boxed and labeled by codon number.

cases or small families, and when investigating small patient cohorts, missense variants may not be interpreted unambiguously. Functional studies of identified missense changes are often time consuming, produce ambiguous results or are not feasible. Therefore, we choose to analyze our data further by using the PANTHER computer program as a complementary approach to differentiate between putative mutations and polymorphisms.

The PANTHER program is a recent development that is used to score sequence variants in silico, and it makes use of the increasing number of database entries regarding homologous and orthologous proteins of human disease 
proteins for evaluating the degree of conservation of amino acid residues, as well as for indicating the potential deleterious effects of missense changes (sub-PSEC scores). ${ }^{18}$ The PANTHER sub-PSEC scores are continuous values from 0 (neutral) to approximately -10 (most likely to be deleterious).

The sub-PSEC score for novel mutations performed using the PANTHER software was in the significant range $(<-3$ $(\mathrm{eg},-7))$ for 3 of the $5 \mathrm{GJB} 1,3$ of the $4 \mathrm{MPZ}$ and for the novel PMP22 mutation, further indicating that at least 7-10 changes have a functional consequence on the protein level (Table 2).

We further evaluated our findings by a PANTHER analysis of published missense variations in these genes (http://www.molgen.ua.ac.be/CMTMutations/default.cfm). All the missense changes described earlier as polymorphisms were scored as such by the PANTHER program (Table 2). However, 28 (68 of 214), 12 (10 of 83) and 18\% (6 of 33) of the published disease-causing mutations and mutations detected in GJB1, MPZ and PMP22, respectively, in this study, showed a sub-PSEC score $>-3(\mathrm{eg},-2)$, that is, these mutations were not classified as disease causing. However, there was a trend apparent that the $P_{\text {deleterious }}$ values were correlated with phenotypic severity, generally suggesting that PANTHER can discriminate between neutral and functional mutants (Table 2). Mutations associated with CMT2, which were thought to result in a loss of function, were less likely to be classified as deleterious.

Further limits of the PANTHER computer program also became apparent as exemplified by the analysis of the
PMP22 mutation, S72L; this mutation was identified in at least 12 independent studies as one of the most severe CMT mutations (leading to $\mathrm{CHN}$ and wheelchair dependency in early childhood and frequently to premature death) among all known CMT-associated genes which contrasts with the PANTHER sub-PSEC score of -2.93 of a disease-unrelated variation. Furthermore, a PANTHER sub-PSEC score of -3.98 was obtained for the T118M PMP22 mutation, which was repeatedly reported in healthy individuals. Other sequence variations might also be misclassified using this line of analysis.

We further investigated whether missense changes, which were not scored as mutations by the PANTHER, were also less supported as such by conventional criteria. We searched all references regarding the MPZ, PMP22 and GJB1 mutations as given in the European CMT Consortium Mutations database for specific information regarding co-segregation within families (number of blood-related individuals), and the number of unrelated controls excluded to harbor the mutation (Table 2). Notably, we found that references contained no information regarding co-segregation within families (number of individuals), and the number of controls excluded to harbor the mutation in up to $60 \%$ of cases (Table 2 ).

For 5/10 (50\%) MPZ, 4/6 (66.7\%) PMP22 and 8/13 (61.5\%) GJB1 variations listed as mutations, but scored as most likely non-deleterious by PANTHER, co-segregation studies were not available or had not been performed, in contrast to $32 / 74$ (43\%) MPZ and 11/27 (40.7\%) PMP22 variants listed as mutations and scored as most likely

Table 2 Newly designed primer sequences for amplification and sequencing of GJB1, MPZ and PMP22

\begin{tabular}{|c|c|c|c|}
\hline \multicolumn{4}{|l|}{ G/B1 } \\
\hline Exon 2 PCR-Primer & $\begin{array}{l}\text { Cx32part1f } \\
\text { Cx32part3r }\end{array}$ & $\begin{array}{l}\text { TGAGGCAGGATGAACTGGACAGGT } \\
\text { TAGCCAGGGAAGGAAGGTTT }\end{array}$ & $990 \mathrm{bp}$ \\
\hline Internal sequencing primer & $\begin{array}{l}\text { Cx32part2f } \\
\text { Cx32part2r }\end{array}$ & $\begin{array}{l}\text { ATCTCCCATGTGCGGCTGTGGTCC } \\
\text { GATGATGAGGTACACСACCT }\end{array}$ & \\
\hline \multicolumn{4}{|l|}{$M P Z$} \\
\hline Exon 1 & $\begin{array}{l}\text { MPZ Ex1f } \\
\text { MPZ Ex1r }\end{array}$ & $\begin{array}{l}\text { CCCGTTCAGTTCCTGGT } \\
\text { GCTGAGAGACACCTGAGTCC }\end{array}$ & $189 \mathrm{bp}$ \\
\hline Exons 2-6 & $\begin{array}{l}\text { MPZ Ex2f } \\
\text { MPZ Ex5/6r }\end{array}$ & $\begin{array}{l}\text { CTTCСТСТGTATСССТTACTG } \\
\text { TAGCTCСATСТCGATGACСA }\end{array}$ & 1694 bp \\
\hline Internal sequencing primer & $\begin{array}{l}\text { MPZ Ex3r } \\
\text { MPZ Ex4f } \\
\text { MPZ Ex4r } \\
\text { MPZ Ex5/6f }\end{array}$ & $\begin{array}{l}\text { GCCTGAATAAAGGTCCTTAGGC } \\
\text { GGAGTCCTACATCCTCAATGCAG } \\
\text { CCCACCCACTGGAGTAGTCTCCG } \\
\text { CAAGGGGAAGAGGAAGCTGT }\end{array}$ & \\
\hline \multicolumn{4}{|l|}{ PMP22 } \\
\hline Exon 1 & $\begin{array}{l}\mathrm{F} \\
\mathrm{R}\end{array}$ & $\begin{array}{l}\text { CTCCTCGCAGGCAGAAACTC } \\
\text { CTGAACCAGCAGGAGCACGGG }\end{array}$ & $202 \mathrm{bp}$ \\
\hline Exon 2 & $\begin{array}{l}\mathrm{F} \\
\mathrm{R}\end{array}$ & $\begin{array}{l}\text { TCAGGATATCTATCTGATTCTC } \\
\text { AAGCTCATGGAGCACAAAACC }\end{array}$ & $156 \mathrm{bp}$ \\
\hline Exon 3 & $\begin{array}{l}\mathrm{F} \\
\mathrm{R}\end{array}$ & $\begin{array}{l}\text { TGGCCAGCTCTCCTAAC } \\
\text { CACСССGСTTCСАСАTG }\end{array}$ & $217 \mathrm{bp}$ \\
\hline Exon 4 & $\begin{array}{l}\mathrm{F} \\
\mathrm{R}\end{array}$ & $\begin{array}{l}\text { GCCATGGACTCTCCGTC } \\
\text { CCTATGTACGCTCAGAG }\end{array}$ & $249 \mathrm{bp}$ \\
\hline
\end{tabular}


deleterious by PANTHER. There were 126/207 (60.9\%) GJB1 mutations with a deleterious PANTHER score without co-segregation studies.

The mechanism by which the MPZ and PMP22 mutations affects protein function remain to be determined in most instances, but may involve a gain of function by aberrant interactions within the MPZ tetramer and within the PMP22 dimer/multimer itself or between MPZ and PMP22, as well as a loss of function by expression of an unstable protein that is rapidly degraded or by an interference with protein translation. These different mechanisms are probably not detected with the same sensitivity, using the approach of evolutionary conservation of residues. However, our analysis suggests that obtaining a sub-PSEC value below the -3 cutoff for sequence variants, at least in the analyzed genes, supports the assumption of a mutation's pathogenicity. We also report that a large number of published MPZ, PMP22 and GJB1 variants have not been evaluated for their pathogenicity. Taken together, our data suggest that at least some of the missense variants contained in the database might represent rare polymorphisms including the MPZ mutation, p.Y33F, and the GJB1 mutation, p.T4K, that are identified in this study.

The frequency of the $17 \mathrm{p} 11.2-\mathrm{p} 12$ duplication in our CMT patient cohort (31.6\%) is in the range of other studies in different ethnical populations. ${ }^{6,7,15,21-26}$ The mutation frequencies of 7.6, 4 and 2\% for GJB1, MPZ and PMP22 mutations, respectively, in our CMT patient cohort were comparable with those reported in a number of other studies. Screening of further known CMT genes is ongoing to identify the genetic causes in the remaining $145 \mathrm{CMT}$ patients.

\section{References}

1 Lupski JR, Garcia CA: Charcot-Marie-Tooth peripheral neurophathies and related disorders; in Scriver CR, Beaudet AL, Sly W, Valle D (eds): The Metabolic and Molecular Bases of Inherited Disease. New York: McGraw-Hill, 2001, pp 5759-5788.

2 Harding AE, Thomas PK: The clinical features of hereditary motor and sensory neuropathy types I and II. Brain 1980; 103: 259-280.

3 Dyck PJ, Lambert EH: Lower motor and primary sensory neuron diseases with peroneal muscular atrophy. I. Neurologic, genetic, and electrophysiologic findings in hereditary polyneuropathies. Arch Neurol 1968; 18: 603-618.

4 Kaku DA, Parry GJ, Malamut R, Lupski JR, Garcia CA: Nerve conduction studies in Charcot-Marie-Tooth polyneuropathy associated with a segmental duplication of chromosome 17. Neurology 1993; 43: 1806-1808.

5 Davis CJ, Bradley WG, Madrid R: The peroneal muscular atrophy syndrome: clinical, genetic, electrophysiological and nerve biopsy studies. I. Clinical, genetic and electrophysiological findings and classification. J Genet Hum 1978; 26: 311-349.

6 Nelis E, Van Broeckhoven C, De Jonghe P et al: Estimation of the mutation frequencies in Charcot-Marie-Tooth disease type 1 and hereditary neuropathy with liability to pressure palsies: a European collaborative study. Eur J Hum Genet 1996; 4: 25-33.

7 Szigeti K, Nelis E, Lupski JR: Molecular diagnostics of CharcotMarie-Tooth disease and related peripheral neuropathies. Neuromolecular Med 2006; 8: 243-254.

8 Shy ME, Jani A, Krajewski K et al: Phenotypic clustering in MPZ mutations. Brain 2004; 127: 371-384.

9 Marrosu MG, Vaccargiu S, Marrosu G, Vannelli A, Cianchetti C, Muntoni F: Charcot-Marie-Tooth disease type 2 associated with mutation of the myelin protein zero gene. Neurology 1998; 50: $1397-1401$.

10 Nelis E, Haites N, Van Broeckhoven C: Mutations in the peripheral myelin genes and associated genes in inherited peripheral neuropathies. Hum Mutat 1999; 13: 11-28.

$11 \mathrm{Mi} \mathrm{H}$, Guo N, Kejariwal A, Thomas PD: PANTHER version 6: protein sequence and function evolution data with expanded representation of biological pathways. Nucleic Acids Res 2007; 35: D247-D252.

12 Badano JL, Inoue K, Katsanis N, Lupski JR: New polymorphic short tandem repeats for PCR-based Charcot-Marie-Tooth disease type 1A duplication diagnosis. Clin Chem 2001; 47: 838-843.

13 Hayasaka K, Nanao K, Tahara $\mathrm{M}$ et al: Isolation and sequence determination of cDNA encoding the major structural protein of human peripheral myelin. Biochem Biophys Res Commun 1991; 180: $515-518$.

14 Floroskufi P, Panas M, Karadima G, Vassilopoulos D: New mutation of the MPZ gene in a family with the Dejerine-Sottas disease phenotype. Muscle Nerve 2007; 35: 667-669.

15 Bort S, Nelis E, Timmerman V et al: Mutational analysis of the MPZ, PMP22 and Cx32 genes in patients of Spanish ancestry with Charcot-Marie-Tooth disease and hereditary neuropathy with liability to pressure palsies. Hum Genet 1997; 99: 746-754.

16 Bone LJ, Deschenes SM, Balice-Gordon RJ, Fischbeck KH, Scherer SS: Connexin32 and X-linked Charcot-Marie-Tooth disease. Neurobiol Dis 1997; 4: 221-230.

17 Shy ME: Peripheral neuropathies caused by mutations in the myelin protein zero. J Neurol Sci 2006; 242: 55-66.

18 Brunham LR, Singaraja RR, Pape TD, Kejariwal A, Thomas PD, Hayden MR: Accurate prediction of the functional significance of single nucleotide polymorphisms and mutations in the ABCA1 gene. PLoS Genet 2005; 1: e83.

19 Nelis E, Holmberg B, Adolfsson R, Holmgren G, van Broeckhoven C: PMP22 Thr(118)Met: recessive CMT1 mutation or polymorphism? Nat Genet 1997; 15: 13-14.

20 Shy ME, Scavina MT, Clark A et al: T118M PMP22 mutation causes partial loss of function and HNPP-like neuropathy. Ann Neurol 2006; 59: 358-364.

21 Silander K, Meretoja P, Juvonen V et al: Spectrum of mutations in Finnish patients with Charcot-Marie-Tooth disease and related neuropathies. Hum Mutat 1998; 12: 59-68.

22 Janssen EA, Kemp S, Hensels GW et al: Connexin32 gene mutations in X-linked dominant Charcot-Marie-Tooth disease (CMTX1). Hum Genet 1997; 99: 501-505.

23 Wise CA, Garcia CA, Davis SN et al: Molecular analyses of unrelated Charcot-Marie-Tooth (CMT) disease patients suggest a high frequency of the CMTIA duplication. Am J Hum Genet 1993; 53: $853-863$.

24 Boerkoel CF, Takashima H, Garcia CA et al: Charcot-Marie-Tooth disease and related neuropathies: mutation distribution and genotype-phenotype correlation. Ann Neurol 2002; 51: 190-201.

25 Mersiyanova IV, Ismailov SM, Polyakov AV et al: Screening for mutations in the peripheral myelin genes PMP22, MPZ and Cx32 (GJB1) in Russian Charcot-Marie-Tooth neuropathy patients. Hum Mutat 2000; 15: 340-347.

26 Choi BO, Lee MS, Shin SH et al: Mutational analysis of PMP22, MPZ, GJB1, EGR2 and NEFL in Korean Charcot-Marie-Tooth neuropathy patients. Hum Mutat 2004; 24: 185-186. 